\title{
Combination of Adaptive Thresh old Algorithm, Watershed and Top-Hat Transform on Image Segmentation Uneven Lighting
}

\author{
Maranto Tua Halomoan ${ }^{1 *}$, Muhammad Zarlis² and Herman Mawengkang ${ }^{2}$ \\ \{maranto@students.usu.ac.id\}
}

\begin{abstract}
${ }^{1}$ Magister of Information Technology, Universitas Sumatera Utara, Medan, Indonesia
${ }^{2}$ Department of Information Technology, Universitas Sumatera Utara, Medan, Indonesia
\end{abstract}

\begin{abstract}
In the previous Saini and Dutta research, there is still a lack of perfect segmentation in the form of window shadows and by examining Cheng and Jun's research, where segmentation on illuminated imagery that has uneven illumination can produce perfect segmentation. In this research, a combination of Adaptive Threshold, Watershed and Top-Hat Transform algorithms is used to segment the image that has uneven lighting. The result of the image segmentation test which has uneven illumination with Adaptive Threshold, Watershed, Top-Hat and Combination algorithm obtained that the smallest value of MSE is in Segmentation result of Combination algorithm and the largest one is in Adaptive Threshold algorithm while the smallest PSNR value is in combination algorithm and the largest is on the Adaptive Threshold algorithm.
\end{abstract}

Keywords: Image Segmentation, Adaptive Threshold, Watershed, Top-Hat Transform.

\section{Introduction}

Image processing process is closely related to the removal or degradation reduction in the image that occurs due to image acquisition process. The degradation includes noise which is an error in pixel value or optical effect such as blur due to a camera that is not in focus or because of camera movement. Image restoration techniques include neighbourhood operations as well as the use of processes in the frequency domain [1].

In Saini and Dutta [8] research conducted that segmentation for uneven lighting image using Adaptive Threshold and Dynamic Window Growing based on incremental window incremental approach. In this proposed method, uneven lighting condition problems have been addressed with the Dynamic Window Growing approach. The algorithm is based on an incremental window increment approach using entropy-based selection criteria. The window specified by the selection criteria is considered as a sub-picture and each sub-image has been segmented by using a minimum deviation standard based on thresholding to improve the result of segmentation. 
In Cheng's research, W. W \& Jun, CX [1] performed image segmentation with uneven illumination by the proposed method based on Homomorphic filtration i.e Top-Hat Transformation and Watershed algorithm. With Homomorphic filtration, it can weaken low frequency components and amplify frequency components high on the frequency domain that makes the image illumination evenly. Top-Hat transformation is adopted to remove a large section of the target image background. The experimental results show that the proposed method is simple and effective that makes uneven illumination image correction achieve satisfactory results. But with a sample of images that have bad adhesion particles, the results of this algorithm process will have border effects and how to improve the adaptability of these algorithms will be the focus of future research.

Based on the research that has been described shows that image segmentation is a very important thing, by dividing an image into homogeneous areas such as color, texture and intensity, it needs an algorithm to change the image representation, therefore this research takes focus on a segmentation problem in which a better approach is required to make uneven illumination images more meaningful and easy to analyze using a combination of adaptive threshold algorithms, top-hat algorithms and watershed transforms.

\section{Methods}

\subsection{Digital Image}

The image is formed from a collection of light intensities arranged in two-dimensional plane. This set of light intensity is expressed in a continuous function $f(x, y)$ where $x$ and $y$ represent the space coordinates and the intensity value of the light gives the color information and the brightness of the image [4].

Digital Image has several formats that have their own characteristic. The format in this digital image is generally based on the type and mode of compression used in the digital image [6].

There are four digital image formats that are often encountered, among others: First Bitmap (BMP), is the most common image format and is a standard format of windows. File size is very large because it can reach the size of megabytes. This file is an uncompressed format and uses the RGB color system (Red, Green,Blue) where each pixel color consists of 3 $\mathrm{R}, \mathrm{G}$, and $\mathrm{B}$ components mixed together.

Furthermore, there is Joint Photographic Expert Group (JPEG / JPG), JPEG format is the most famous format until now. This is because of its small size (only tens / hundreds of KB only), and is portable. This file format is often used in the field of photography to store image files from analog recording to digital converter (ADC)

The three GIF (Graphics Interchange Format) formats, GIF files allow the addition of transparent colors and can be used to create simple animations, but currently the GIF standard is only 256 colors maximum.

Lastly PNG (Portable Network Graphics), PNG-formatted Image was developed as another alternative to GIF, which uses patents from LZW-compression algorithms. PNG is an excellent image format for internet graphics, as it supports transparency within browser and has its own beauty that can not be shown GIF or even JPG

\subsection{Image Enhancement}


The operations performed to transform an image into another image can be categorized based on the purpose of transformation as well as the scope of operation performed on the image [6]. Based on the purpose of image processing operations transformation categorized as follows: 1. Improved Image Quality (Image Enhancement) Image enhancement operations aim to improve certain features of the imagery. 2. Image Restoration: The image recovery operation aims to restore the image condition to a previously known condition due to an interruption that causes image degradation.

Especially for image repair methods with image binarization methods, J. Sauvola et al [5] proposes an adaptive binarization method that can separate well the text, background, and image components of a document image. With the determination of the local threshold value, Sauvola successfully overcome the main problem of document image damage caused by noise and different illumination levels. A review of the comparison of different types of thresholding algorithms to separate the text and background portions of a complex document is given by G. Leedham et al [2].

Graham provides a comparison review for five algorithm: The Niblack algorithm, the Mean Gradient technique, the background substraction method, the QIR (Quadratic Integral Ratio) method, and the Yanowitz and Bruckstein methods. To improve the performance, it should doa combination of algorithms according to different document types. Surveys of thresholding techniques on images with quantitative performance evaluations were also performed by M. Sezgin et al [7].

Before doing image processing, firstly done the reading and calculation of Pixel value. The pixel value reading is performed on each color component (RGB) using the formula:

Value of $\mathrm{R}=\mathrm{c}$ and 255

Value of $\mathrm{G}=(\mathrm{c}$ and 65,280$) / 256$

Value of $\mathrm{B}=((\mathrm{c}$ and $16,711,680) / 256) / 25$

\subsection{Calculating Grayscale Image Value}

The color image matrix is transformed into grayscale image by calculating the average colors of Red, Green and Blue. Mathematically the calculation is as follows.

$\mathrm{f}_{0}(\mathrm{x}, \mathrm{y})=\left(\frac{\mathrm{f}^{\mathrm{R}}(x, y)+\mathrm{f}^{\mathrm{G}}(x, y)+\mathrm{f}^{\mathrm{B}}(x, y)}{3}\right)$

\subsection{Adaptive Threshold Algorithm}

Thesholding is done by dividing the image using multiple sub-images. Then in each sub image, segmentation is done by using different threshold [9].

In the Adaptive Thresholding method, the threshold value of the image can be calculated by the following formula:

$\mathrm{T}=\frac{\sum_{(x, y)} \sum \in W f(x, y)}{N_{W}}-C$ 
Where,

- W is a processed block,

- NW is the number of pixels in each block W,

- $\mathrm{C}$ is a constant which can be determined freely, if $\mathrm{C}=0$, then the threshold value is equal to the mean of each pixel in the block.

The AdaptiveThresholding process with adaptive window selection based on sharpness level measurement. Here are the steps:

1. Digital image input.

2. Divide the image into sub images according to the window size.

3. Find the image sharpness value on eachwindow (sub image).

4. Create a feature $f^{\prime}(x, y)$, where the window is pixel and the image sharpness value is as a result of its value.

5. Calculate the value of thresholdT 'with value $\mathrm{f}^{\prime}(\mathrm{x}, \mathrm{y})$ as the pixel value of the image.

6. Pixel intensity segmentation in a window (sub image) that has a sharpness value $>\mathrm{T}$ '.

7. Check if the whole window (subcitra) has been segmented. If yes, then stop.

8. Find and save the number of windows that have an image value $\leq \mathrm{T}$ '.

9. Save the number of window values $\leq \mathrm{T}$ 'to a variable that is the number ofwindows.

10. Create and initialize variables $i=0$.

11. Grow and calculate the value of sharpness level in window (i).

12. Check if the window size (i)> sizeimage. If yes, then go to step 14.

13. Check if the sharpness of the image window (i) $\leq \mathrm{T}$ '. If yes, then go to step 11 .

14. Pixel intensity segmentation in window (i).

15. Increment $i$.

16. Check if $\mathrm{i} \leq$ jumwindow. If yes, then go to step xi.

17. Do a merge window (sub image), so it becomes a complete image.

18. Stop

\subsection{Watershed Algorithm}

Watershed is one of the methods used for image segmentation. The concept contained in Watershed is able to visualize an image in three 3D dimensions.

Below are the steps of the Watershed algorithm.

i. Determine the region (region) by representing the object and the background that has a value of 0 .

ii. Conduct a dilation on the area using the $3 \times 3$ element structure.

iii. Form the dam at the position where the two areas are connected.

iv. Repeat step (3) until all areas are joined.

Considered M1, M2, M3, .., MR is a collection of point coordinates within a regional minimum of a picture $\mathrm{g}(\mathrm{x}, \mathrm{y})$. There is a $\mathrm{C}(\mathrm{Mi})$ which is a collection of coordinates on the catchmentbasin and corresponds to the minimum area Mi. Min and max notation is used to mark the minimum value and maximum value of $\mathrm{g}(\mathrm{x}, \mathrm{y})$.

Then it is considered $T[n]$ is the set of coordinates $(s, t)$ where $g(s, t)<n$, so it can be defined:

$\mathrm{T}[\mathrm{n}]=\{(\mathrm{s}, \mathrm{t}) \mid \mathrm{g}(\mathrm{x}, \mathrm{y})<\mathrm{n}\}$

Geometrically, $\mathrm{T}[\mathrm{n}]$ is the set of coordinates of the point located at $\mathrm{g}(\mathrm{x}, \mathrm{y})$ and lies below the plane of $\mathrm{g}(\mathrm{x}, \mathrm{y})=\mathrm{n}$. The topography will be flowed with an integer addition starting from 
$\mathrm{n}=\min +1$ to $\mathrm{n}=\max +1$. At each addition of $\mathrm{n}$, the algorithm needs to know the number of points that are below the flow depth. Generally, the area under $\mathrm{g}(\mathrm{x}, \mathrm{y})=\mathrm{n}$ is given a black or a value 0 and above it is white or a value of 1 . Then it is assumed $\mathrm{Cn}$ (Mi) is the set of point coordinates in the basin catchment corresponding to the minimum Mi which is included in the stage $\mathrm{n}$. $\mathrm{Cn}(\mathrm{Mi})$ can be seen as a binary image by using the equation:

$$
C_{n}\left(M_{i}\right)=C\left(M_{i}\right) \cap T[n]
$$

In other words $\mathrm{Cn}(\mathrm{Mi})=1$ lies in the location $(\mathrm{x}, \mathrm{y})$ if $(\mathrm{x}, \mathrm{y}) \in \mathrm{C}(\mathrm{Mi})$ and $(\mathrm{x}, \mathrm{y}) \in \mathrm{T}[\mathrm{n}]$, besides that the value of $\mathrm{Cn}(\mathrm{Mi})=0$. Next, it is assumed that $\mathrm{C}[\mathrm{n}]$ is a composite of the flow in the catchmentbasin at stage $\mathrm{n}$ :

$$
C[n]=\bigcup_{i=1}^{R} \mathrm{C}_{\mathrm{n}}\left(M_{i}\right)
$$

and $\mathrm{C}[\max +1]$ is a composite of all basin catchments:

$C$ [n-1] is a subset of $C[n]$ and $C[n]$ is a subset of $T[n]$ then $C[n-1]$ is a subset of $T$ [n]. From here it is found that each connected component of $C[n-1]$ is present on one connected component of $\mathrm{T}[\mathrm{n}]$. The algorithm for finding the first watershed line is initialized with $\mathrm{C}$ $[\min +1]=\mathrm{T}[\min +1]$.

The algorithm will be processed recursively with the assumption at stage $n$ then $C$ [n-1] has been formed. The procedure for obtaining $\mathrm{C}[\mathrm{n}]$ from $\mathrm{C}[\mathrm{n}-1]$ is as follows. Assume $\mathrm{Q}$ is a collection of connected components in $T$ [n]. Thus for each component connected to $Q \in Q$ [n], there are three possibilities:

a. $\mathrm{q} \cap \mathrm{C}[\mathrm{n}-1]$ is empty

b. $\mathrm{q} \cap \mathrm{C}[\mathrm{n}-1]$ has 1 connected component of $\mathrm{C}[\mathrm{n}-1]$

c. $\mathrm{q} \cap \mathrm{C}[\mathrm{n}-1]$ has more than 1 component connected from $C[\mathrm{n}-1]$

\subsection{Top-Hat Transform Algorithm}

Top-hat transformation is the result of input image subtraction with image that has undergone operation opening i.e difference between original image and image after experiencing opening operation.

Combining measurements with an opening account will result in Top-hat transform. Tophat Transformation gray scale image $\mathrm{f}$ is defined as the least of the opening:

That $(f)=f-\left(\begin{array}{lll}f & 0\end{array}\right)$

Top-hat transformation is used for bright objects in dark backgrounds. An important use of top-hat transformation is to improve the effect of uniform illumination, because good illumination plays an important role in the process of extracting objects from the background. This process, called segmentation, is one of the first steps to be done in automated image analysis. The opening process serves to eliminate small objects but retains their original size. Opening process is a processerosion with dilation image.

$F(x, y)$ o $S E=(f(x, y) \theta S E) \Theta S E$ 


\subsection{Combination algorithm}

The combination algorithm is acombination of image segmentation algorithmsamongAdaptiveThresholding, Watershed and Top-Hat algorithms where AdaptiveThresholdingsegmentation image is reprocessed with Watershed Transformation algorithms as well as Top-Hat to obtain a clearer image as a result of a combination transformation. The Combination algorithm steps can be seen in points 2.4 to 2.6.

\subsection{Data Set}

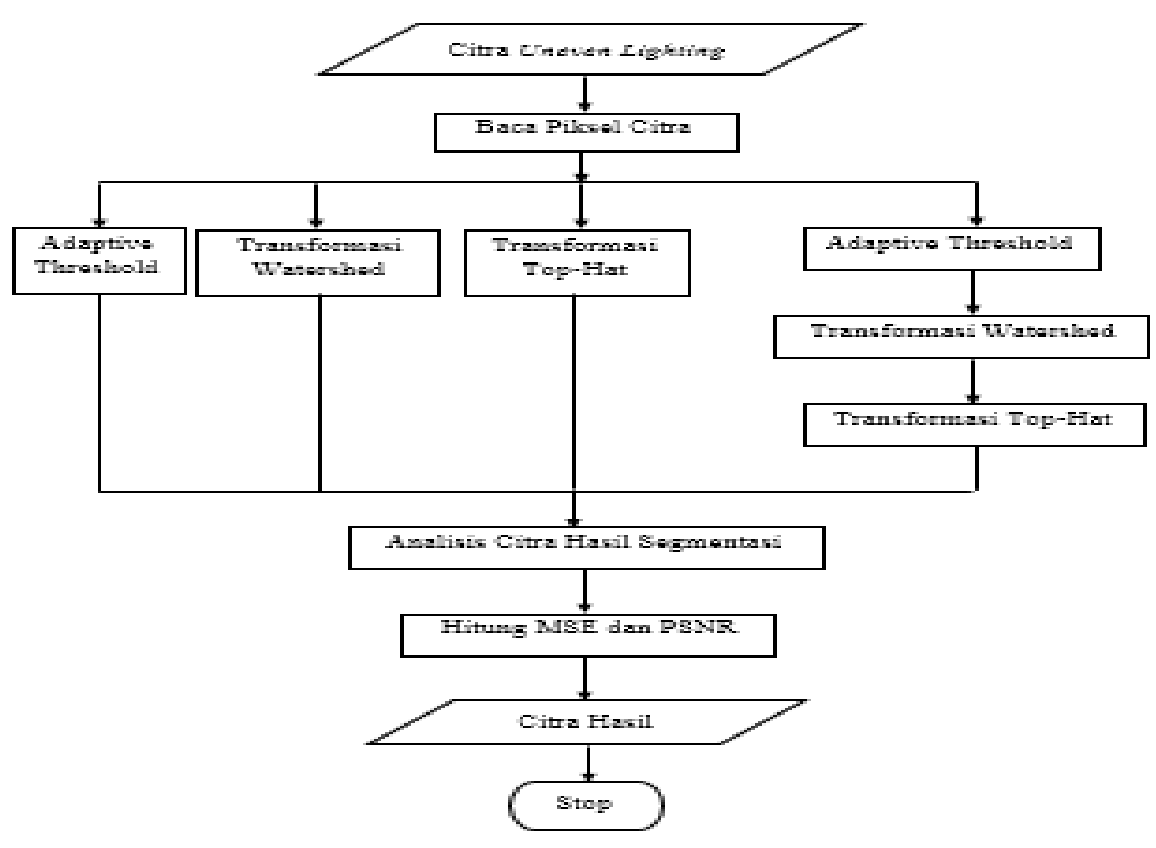

Figure.1. Research Framework

\subsection{Research Framework}

In this research the original image in the form of uneven lighting image is done by reading the value of pixel value for each image area. The process of segmenting the Adaptive Thresholding algorithm is done by forming a window whose size is adjusted to its threshold value. In segmentation with Watershed transformation algorithm and Top Hat, segmentation is done by performing calculation of pixel value by Watershed and Top Hat transformation. In segmentation with the combination algorithm is segmentation is done by Adaptive Thresholding algorithm and Watershed transformation as well as Top Hat. The final stage is done by image analysis of the results of segmentation with the calculation of the value of MSE and PSNR and by comparing the original image with the image of the visual segmentation results. 


\section{Result}

The results of segmentation testing with Threshold Adaptive algorithm is a test with image dimension $400 \times 300$ pixels that produce the value of MSE, PSNR and image of the results of its segmentation. Value of MSE and PSNR Image Segmentation Threshold Adaptive Table.1:Value MSE and PSNR ThresholdAdaptive Segmentation

\begin{tabular}{|c|c|c|c|c|}
\hline \multirow{2}{*}{ No. } & \multirow{2}{*}{ File Cover } & \multirow{2}{*}{ Size (Kbyte) } & \multicolumn{2}{|c|}{ Parameter } \\
\hline & & & MSE & PSNR \\
\hline 1 & Image-1 & 9.6806 & 6998.6882 & 9.6806 \\
\hline 2 & Image-2 & 13.5094 & 2898.2498 & 13.5094 \\
\hline 3 & Image-3 & 11.0306 & 5128.9004 & 11.0306 \\
\hline 4 & Image-4 & 18.2266 & 978.1784 & 18.2266 \\
\hline 5 & Image-5 & 8.3547 & 9497.5385 & 8.3547 \\
\hline 6 & Image-6 & 13.3592 & 3000.2876 & 13.3592 \\
\hline 7 & Image-7 & 19.0384 & 811.4112 & 19.0384 \\
\hline 8 & Image-8 & 16.7968 & 1359.557 & 16.7968 \\
\hline 9 & Image-9 & 16.2132 & 1555.1164 & 16.2132 \\
\hline 10 & Image-10 & 11.8712 & 4226.3037 & 11.8712 \\
\hline \multicolumn{3}{|c|}{ Average } & 3645.4231 & 13.8080 \\
\hline
\end{tabular}

Table.2. Value MSE and PSNR The Result of Watershed Segmentation

\begin{tabular}{|c|c|c|c|c|}
\hline \multirow{2}{*}{ No } & \multirow{2}{*}{ File Cover } & \multirow{2}{*}{ Size (Kbyte) } & \multicolumn{2}{|c|}{ Parameter } \\
\cline { 3 - 5 } & & & MSE & PSNR \\
\hline 1 & Image-1 & 158 & 1203.6833 & 17.3258 \\
\hline 2 & Image-2 & 25.6 & 2226.5365 & 14.6545 \\
\hline 3 & Image-3 & 45.8 & 644.9799 & 20.0353 \\
\hline 4 & Image-4 & 89.5 & 1229.8864 & 17.2322 \\
\hline 5 & Image-5 & 46.3 & 1245.3107 & 17.1781 \\
\hline 6 & Image-6 & 31.2 & 859.4761 & 18.7877 \\
\hline 7 & Image-7 & 34.5 & 2763.7301 & 13.7159 \\
\hline 8 & Image-8 & 42.5 & 6498.3365 & 10.0028 \\
\hline 9 & Image-9 & 33.1 & 431.5203 & 21.7852 \\
\hline 10 & Image-10 & 41.4 & 1246.7857 & 17.1729 \\
\hline & Average & & $\mathbf{1 8 3 5 . 0 2 4 6}$ & $\mathbf{1 6 . 7 8 9 0}$ \\
\hline
\end{tabular}


Table.3: Value MSE and PSNR The Result of Top-Hat Segmentation

\begin{tabular}{|c|c|c|c|c|}
\hline \multirow{2}{*}{ No } & \multirow{2}{*}{ File Cover } & \multirow{2}{*}{ Size (Kbyte) } & \multicolumn{2}{|c|}{ Parameter } \\
\cline { 3 - 5 } & & & MSE & PSNR \\
\hline 1 & Image-1 & 158 & 4830.1963 & 11.2912 \\
\hline 2 & Image-2 & 25.6 & 2319.7229 & 14.4764 \\
\hline 3 & Image-3 & 45.8 & 3664.324 & 12.4904 \\
\hline 4 & Image-4 & 89.5 & 67.9101 & 29.8115 \\
\hline 5 & Image-5 & 46.3 & 7698.0512 & 9.2670 \\
\hline 6 & Image-6 & 31.2 & 2011.0876 & 15.0965 \\
\hline 7 & Image-7 & 34.5 & 112.8382 & 27.6062 \\
\hline 8 & Image-8 & 42.5 & 185.6022 & 25.4450 \\
\hline 9 & Image-9 & 33.1 & 765.4475 & 19.2916 \\
\hline 10 & Image-10 & 41.4 & 2859.885 & 13.5673 \\
\hline & Average & & $\mathbf{2 4 5 1 . 5 0 6 5}$ & $\mathbf{1 7 . 8 3 4 3}$ \\
\hline
\end{tabular}

Table.4: The Result of Combination Segmentation

\begin{tabular}{|c|c|c|c|l|}
\hline \multirow{2}{*}{ No } & \multirow{2}{*}{ File Cover } & \multirow{2}{*}{ Size (Kbyte) } & \multicolumn{2}{|c|}{ Parameter } \\
\cline { 3 - 5 } & & & MSE & PSNR \\
\hline 1 & Image-1 & 158 & 1182.622 & 17.4024 \\
\hline 2 & Image-2 & 25.6 & 487.6495 & 21.2497 \\
\hline 3 & Image-3 & 45.8 & 901.159 & 18.5828 \\
\hline 4 & Image-4 & 89.5 & 154.6979 & 26.2359 \\
\hline 5 & Image-5 & 46.3 & 1674.2872 & 15.8925 \\
\hline 6 & Image-6 & 31.2 & 488.6608 & 21.2401 \\
\hline 7 & Image-7 & 34.5 & 158.8865 & 26.1200 \\
\hline 8 & Image-8 & 42.5 & 229.0542 & 24.5314 \\
\hline 9 & Image-9 & 33.1 & 273.9042 & 23.7563 \\
\hline 10 & Image-10 & 41.4 & 712.4302 & 19.6034 \\
\hline & Average & & $\mathbf{6 2 6 . 3 3 5 2}$ & $\mathbf{2 1 . 4 6 1 4}$ \\
\hline
\end{tabular}

\section{Discussion}


The segmentation test is performed on 10 image color images with Adaptive Threshold, Watershed, Top-Hat and Combination algorithms, the result is shown in Table.5

Table.5. The Result ofSegmentation Algorithm Adaptive Threshold, Watershed, Top-Hat and Combination

\begin{tabular}{|c|l|c|c|}
\hline No & \multicolumn{1}{|c|}{ Algorithm } & Average MSE & Average PSNR \\
\hline 1 & Adaptive Threshold & 3645.4231 & 13.8081 \\
\hline 2 & Watershed & 1835.0246 & 16.7891 \\
\hline 3 & Top-Hat & 2451.5065 & 17.8343 \\
\hline 4 & Combination & 626.3348 & 21.4614 \\
\hline
\end{tabular}

From Table.5 Adaptive threshold, watershed, top-hat segmentation and combinations for unevenly illuminated images are obtained that the smallest MSE values exist in the combination algorithm segmentation and the largest in the adaptive threshold algorithm, the smallest PSNR value in the adaptive threshold algorithm and the largest on the combination algorithm.

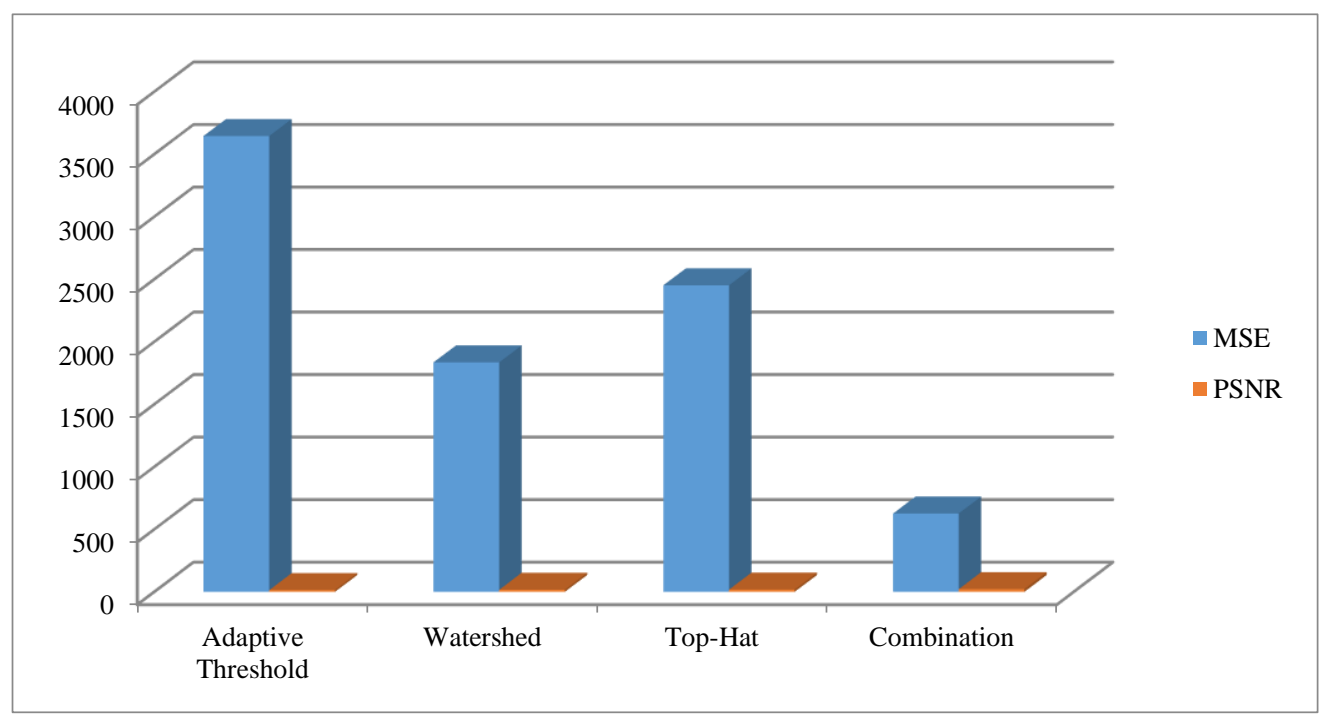

Figure.2. Graphic of The Result ofSegmentation Algorithm Adaptive Threshold, Watershed, Top-Hat and Combination.

\section{Conclusion}


From the results of image segmentation testing with Adaptive Threshold, Watershed, TopHat and combination it can be seen that the combination method is able to segment the image that has uneven illumination well. It is seen that in the image that has been combined better segmentation than the image of Adaptive Threshold and Watershed and Top-Hat combined image. This can be seen also by decreasing the value of MSE and increasing the value of PSNR in the combination algorithm. The decreasing value of MSE and the increasing value of PSNR shows that the segmentation in an image is getting better.

\section{References}

[1] Cheng, W. W \& Jun, C. X. 2013. A Segmentation Method for Uneven Illumination Particle Images. Research Journal of Applied Sciences, Engineering and Technology 2013. Department of Information and Control, Weifang University,Weifang, 261061, China.

[2] G.Leedham. 2003. Comparison of Some Thresholding Algorithms for Text/Background Segmentation in Difficult Document Images. School of Computer Engineering, Nanyang Technological University Nanyang Avenue, Singapore

[3] Gonzalez, R.C. \& Woods, R.E. Digital Image Processing. Second edition, USA: Addison-Wesley Publishing Co, University of Tennessee.

[4] Gonzalez, R.C. \& Woods, R.E. Digital Image Processing. Third edition, USA: AddisonWesley Publishing Co, University of Tennessee.

[5] J.Sauvola \& M. PietikaKinen, C. 1999. Adaptive Document Image Binarization, Machine Vision and Media Processing Group, Infotech Oulu, University of Oulu, Finland

[6] Kambam Bijen Singh et al. 2017. Image Enhancement with the Application of Local and Global Enhancement Methods for Dark Images. National Institute of Technology Meghalaya Shillong- India.

[7] M.Sezgin et al 2004. Survey Over Image Thresholding Techniques And Quantitative Performance Evaluation. Journal of Electronic Imaging

[8] Saini, R. \& Dutta, M. 2012. Image Segmentation for Uneven Lighting Images using Adaptive Thresholding and Dynamic Window based on Incremental Window Growing Approach. International Journal of Computer Applications (0975 - 8887) Volume 56No.13, October 2012. National Institute of Technical Teachers Training and Research, (NITTTR) Chandigarh, 160019, India.

[9] Samanta, D \& Sanyal, G. 2011. Development of Adaptive Thresholding Technique for Classification of Synthetic Aperture Radar Images. International Journal of Computer Science \& Technology. Dept. of CSE, National Institute of Technology, Durgapur, West Bengal, India.

[10] Yang, J \& Miao, Z. 2016. On the Image Enhancement Histogram Processing. 2016 3rd International Conference on Informative and Cybernetics for Computational Social Systems (ICCSS). School Electronics and Information Engineering. Liaoning University of Technology China. 
[11] Yelmanova, E \& Romanyshyn, Y. 2017. Histogram-based Method for Image Contrast Enhancement. Department of ECT, Lviv Polytechnic National University, UKRAINE, University of Warmia and Mazury in Olsztyn, Poland.

[12] Zhao, J., Wang, H., Zhang, L. \& Wang, C. 2015. The Method of Flotation Froth Image Segmentation Based on Threshold Level Set. Department of Software Engineering, University of Science and Technology Liaoning, Anshan, China 